\title{
Guar gum/borax hydrogel: Rheological, low field NMR and release characterizations
}

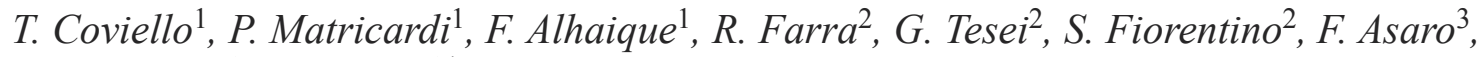 \\ G. Milcovich ${ }^{3}$, M. Grassi ${ }^{2}$ \\ ${ }^{1}$ Department of Drug Chemistry and Technologies, 'Sapienza', University of Rome, Piazzale Aldo Moro 5, 00185 Rome, \\ Italy \\ ${ }^{2}$ Department of Engineering and Architecture, University of Trieste, Piazzale Europa 1, 34127 Trieste, Italy \\ ${ }^{3}$ Department of Chemical and Pharmaceutical Sciences, University of Trieste, Via Giorgeri 1, 34127 Trieste, Italy
}

Received 26 March 2013; accepted in revised form 19 May 2013

\begin{abstract}
Guar gum (GG) and Guar gum/borax (GGb) hydrogels are studied by means of rheology, Low Field Nuclear Magnetic Resonance (LF NMR) and model drug release tests. These three approaches are used to estimate the mesh size ( $\xi$ ) of the polymeric network. A comparison with similar Scleroglucan systems is carried out. In the case of GGb, the rheological and Low Field NMR estimations of $\xi$ lead to comparable results, while the drug release approach seems to underestimate $\xi$. Such discrepancy is attributed to the viscous effect of some polymeric chains that, although bound to the network to one end, can freely fluctuate among meshes. The viscous drag exerted by these chains slows down drug diffusion through the polymeric network. A proof for this hypothesis is given by the case of Scleroglucan gel, where the viscous contribution is not so significant and a good agreement between the rheological and release test approaches was found.
\end{abstract}

Keywords: polymer gels, rheology, low field NMR, transport processes, mesh-size

\section{Introduction}

Guar gum (GG) is a neutral galactomannan, extracted from the seeds of Cyamopsis tetragonoloba. It consists of a linear backbone of $\beta(1 \rightarrow 4)$-linked Dmannopyranose units (Man) and with the presence of randomly attached $\alpha(1 \rightarrow 6)$-linked galactopyranose units (Gal) as side chains [1]. Due to the presence of these galactose units, the polymer is soluble in water [2]. The ratio of mannose to galactose units $(M / G)$ depends on climate variations and ranges from 1.5:1 to 1.8:1. Because of its low cost and its ability to produce a highly viscous solution even at low concentrations, GG finds important applications in food [3], in oil recovery [4] and in personal care industries [5]. The high viscosity of GG solutions arises from the high molecular weight of GG (up to 2 million and further) [6] and from the presence of extensive intermolecular associations (entanglements) by means of hydrogen bonds.

In aqueous solution GG assumes a flexible coil conformation as evidenced by the Mark-HouwinkSakurada exponent and by the relatively low value of its characteristic ratio and its persistence length [7]. GG, crosslinked with glutaraldehyde, was proposed for colon delivery [8], and it was also tested as a matrix for oral solid dosage forms [9].

Scleroglucan (SCLG), a water soluble polysaccharide produced by fungi of the genus Sclerotium, consists of a main chain of $(1 \rightarrow 3)$-linked $\beta$-D-glucopyranosyl units bearing, every third unit, a single $\beta$-D-glucopyranosyl unit linked $(1 \rightarrow 6)$. It is known that SCLG assumes a triple-stranded helical confor-

\footnotetext{
${ }^{*}$ Corresponding author, e-mail: mariog@dicamp.univ.trieste.it (c) BME-PT
} 
mation in aqueous solution and a single coiled disordered conformation in methylsulphoxide or at high $\mathrm{pH}$ values $(\mathrm{NaOH}>0.2 \mathrm{M})[10,11]$. Due to its peculiar properties, SCLG was extensively used for various commercial applications (secondary oil recovery, ceramic glazes, food, paints, cosmetics, etc.) [12] and it was also investigated for modified/ sustained release formulations and ophthalmic preparations [13].

Actually, it is well known that borax is an efficient crosslinker for polymers bearing hydroxyl groups but the type of formed linkages is still debated and so far two main models have been proposed. The most popular one implies the existence of pure chemical crosslinks between the polymeric chains and borax [14], and it was proposed for the GG/ borax interactions. According to the other model, the borax ions hold together the polymeric chains by means of mixed physical/chemical linkages. This model was firstly proposed for poly-(vinylalcohol) [15] and it was recently suggested also for SCLG $[16,17]$.

The considerable effort devoted to the study of polymer-ion complexes is due to the wide range of application of these systems. In particular, the complex between GG and borate was previously studied by several authors $[18,19]$ that investigated the effect of polymer and borate concentration, temperature, environmental $\mathrm{pH}$ conditions, and GG molecular weight on the peculiar rheological properties detected by the frequency dependence of relaxation spectra. Furthermore, a detailed study of the crosslinking reaction of borate ion with polyhydroxy polymers was carried out by means of ${ }^{11} \mathrm{~B}$ NMR spectroscopy on dilute mixtures of borate ions and GG, for the acquisition of further insight into the complexation mechanism. Thus, the values of complexation equilibrium constants and the complexation enthalpy at various measuring conditions were calculated [14, 20-22]. In addition, specific investigations on the rheological properties of the schizophyllan/borate system were reported (schizophyllan has the same repeating unit of SCLG) in other papers $[23,24]$. It is interesting to note that both, GG and SCLG, interact with borax leading to a three-dimensional network that, besides the intrinsic differences between the two polymers (see below), shows a peculiar anisotropic elongation during swelling, when tablets of these two systems are prepared. In particular, GG and SCLG show important different characteristics: (a) GG dissolves in water as a random coil while SCLG exhibits a triple helix conformation in aqueous solution with a persistence length of about $200 \mathrm{~nm}[10,25]$; (b) borax promotes a rapid gelation of GG [26] by means of crosslinks characterized by a lifetime of the order of seconds [27], leading to self-healing properties of the network. On the other side, SCLG requires several hours for gelation in the presence of borax and no self-healing occurs. Nevertheless, both, GG and SCLG, in the presence of borax, are capable to give self-sustaining gels [28]. Furthermore, also the interaction with borax takes place in a different way. In the case of SCLG the borax promotes mixed (chemical and physical) interactions between triplexes; on the other side, in the case of GG the borax forms chemical bridges between chains by means of reversible linkages. In addition, molecular dynamics simulation and AFM images indicate that the borax groups increase the stiffness of GG making such system more similar to SCLG/borax (SCLGb). During the swelling the labile nature of the borax cross-links in the GG makes the interchain interactions able to undergo the needed rearrangement, similar to that of SCLG where full chemical bridges are not present. These similarities explain the quite unexpected parallel swelling behaviour of GG and SCLG, in the presence of borax $[16,28]$.

However, in all these previous research works, no attention was specifically focused on the estimation of the mesh sizes of the networks, a very important parameter for the numerous implications that it may have on these hydrogel systems when used for industrial and/or pharmaceutical applications.

In this frame, aim of the present work was to characterise $\mathrm{GG}$ and $\mathrm{GG} /$ borax (GGb) systems resorting to three different approaches, rheology, Low Field NMR (LF NMR) and release tests. In fact, the joint use of these approaches provides, for the first time at our best knowledge, more complete information about the polymeric chain architectures (polymeric networks), also in terms of mesh size estimation, and their possible modifications with temperature in physiological conditions ( 25 and $37^{\circ} \mathrm{C}$ ). In addition, the characteristics of GG and GGb systems were compared with those of the SCLG and SCLGb systems. 


\section{Experimental section \\ 2.1. Materials}

Guar Gum (GG) was provided by CarboMer (San Diego, USA). The ratio between mannose and galactose was estimated by means of ${ }^{1} \mathrm{H}$ NMR (carried out at $70^{\circ} \mathrm{C}$ with a Brucker AVANCE AQS 600 spectrometer, operating at $600.13 \mathrm{MHz}$ ) and an $M / G$ value of $\approx 1.5$ was found. The molecular weight $\left(1.2 \cdot 10^{6}\right)$ was estimated by means of viscometric measurements carried out at $25^{\circ} \mathrm{C}$. Scleroglucan (SCLG) was provided by Degussa (Germany). The molecular weight $\left(1.1 \cdot 10^{6}\right)$ was estimated by means of viscometric measurements carried out at $25^{\circ} \mathrm{C}$ in $0.01 \mathrm{M} \mathrm{NaOH}$. For the viscosity measurements, an automatic viscometer (Instrument Schott AVS 370, Lauda, Germany) with a water bath (Lauda $0.15 \mathrm{~T}$ ) allowing the temperature control to $0.1^{\circ} \mathrm{C}$ was used. An Ubbelohde capillary viscometer (Type No 531 01 , with a capillary diameter $=0.54 \mathrm{~mm}$, SchottGeräte) for dilution sequences was used. The GG solutions were prepared in distilled water while the SCLG solutions were prepared in $0.01 \mathrm{~N} \mathrm{NaOH}$ (in order to break possible aggregates). Before measurements, the samples were filtered twice with $1.2 \mu$ Millipore filters. From the flux time of solvent and solutions the intrinsic viscosity, $[\eta]\left[\mathrm{cm}^{3} / \mathrm{g}\right]$, was estimated for each polymer. According to the MarkHouwink-Sakurada equation, the intrinsic viscosity is related to the molar mass of the sample: $[\eta]=$ $K M_{\mathrm{w}}{ }^{\mathrm{a}}$, where $K$ and $a$ are constants for each polymer-solvent system at a given temperature. From the value of $K$ and $a$ found in the literature for the GG [11] and for the SCLG [7] samples, the molecular weight of the two polymers were evaluated. Theophylline (TPH, molecular weight 198) and borax were Carlo Erba products (Italy), Vitamin $\mathrm{B}_{12}$ (Vit $B_{12}$, molecular weight 13 500) was purchased from Fluka (Germany). All other products and reagents were of analytical grade. Distilled water was always used.

\subsection{Polymer purification}

A given amount of polymer (GG and SCLG) was dissolved in distilled water (polymer concentration, $c_{\mathrm{p}}=0.5 \% \mathrm{w} / \mathrm{v}$ ). GG samples were kept under magnetic and mechanical stirring at $60^{\circ} \mathrm{C}$ for $24 \mathrm{~h}$ and then at room temperature for 24 additional hours [29]. SCLG samples were kept under magnetic and mechanical stirring for $24 \mathrm{~h}$ at room temperature. The resulting solutions were exhaustively dialysed at $7{ }^{\circ} \mathrm{C}$ against distilled water with dialysis membranes of a cut-off 12 000-14 000 and then freeze dried. The lyophilized products were stored in a desiccator until use.

\subsection{Hydrogel preparations}

A given amount of GG or SCLG (200 mg for the release experiments and $35 \mathrm{mg}$ for the rheological analysis and for NMR measurements; $c_{\mathrm{p}}=0.7 \% \mathrm{w} / \mathrm{v}$ ) was dissolved in water for $24 \mathrm{~h}$. GG and SCLG crosslinking was carried out by addition of $0.1 \mathrm{M}$ borax solution to the homogeneous polymer system in order to get a unitary value of the ratio between borax moles and moles of the repeating GG or SCLG units $(r=1)$. The resulting mixture was magnetically stirred for $5 \mathrm{~min}$ and then left for 2 days at $7^{\circ} \mathrm{C}$ for gel setting. For the release experiments, a known amount of model drug was first dissolved in water before the addition of the polymer.

\subsection{Rheological characterization}

The rheological characterization, carried out at 25 and $37^{\circ} \mathrm{C}$, was performed by means of a controlled stress rheometer, Haake Rheo-Stress RS300 model, with a Thermo Haake DC50 water bath. Two geometries were used: a cone-plate device (C60/1 Ti with a cone diameter of $60 \mathrm{~mm}$ and a cone angle of $1^{\circ}$ and a MP60 steel 8/800 plate with a diameter of $60 \mathrm{~mm}$, Haake) for the GG and SCLG samples and a grained plate-plate device (Haake PP35/S: diameter $=35 \mathrm{~mm}$; gap between plates $=1 \mathrm{~mm}$ ) was used for the SCLGb and GGb samples in order to prevent wall slippage phenomena [30]. To perform the measurements on SCLGb and GGb, the hydrogels, obtained with a thickness of $1.0-3.0 \mathrm{~mm}$, were removed with the aid of a small spatula from the beaker in which they had settled, and they were laid with care on the lower plate of the rheometer. The upper plate was then lowered until it reached the hydrogel surface. Gap-setting optimizations were undertaken according to a procedure described elsewhere [31]. When GG or SCLG were tested, an appropriate amount of the samples was spread onto the plate geometry to obtain a sample of the appropriate height. To avoid gel shrinking due to a possible solvent evaporation, the equipment was kept inside a glass bell with a constant moisture level. Rheological properties were studied by means of oscillatory tests. In particular, the hydrogel linear viscoelastic regions were assessed, at $1 \mathrm{~Hz}$, by stress 
sweep experiments. Frequency sweep tests were carried out in the frequency $(f)$ range $0.01-100 \mathrm{~Hz}$ at constant deformations $\gamma=0.01$ (well inside the linear viscoelastic range for all the studied hydrogels). Each test was carried out in duplicate.

The generalized Maxwell model [30] was used for the theoretical dependence of the elastic $\left(G^{\prime}\right)$ and viscous $\left(G^{\prime \prime}\right)$ moduli on pulsation $\omega=2 \pi f(f=$ solicitation frequency) (Equations (1) and (2)):

$$
\begin{aligned}
G^{\prime} & =\sum_{\mathrm{i}=1}^{\mathrm{n}} G_{\mathrm{i}} \frac{\left(\lambda_{\mathrm{i}} \omega\right)^{2}}{1+\left(\lambda_{\mathrm{i}} \omega\right)^{2}}, \quad G_{\mathrm{i}}=\frac{\eta_{\mathrm{i}}}{\lambda_{\mathrm{i}}} \\
G^{\prime \prime} & =\sum_{\mathrm{i}=1}^{\mathrm{n}} G_{\mathrm{i}} \frac{\omega \lambda_{\mathrm{i}}}{1+\left(\lambda_{\mathrm{i}} \omega\right)^{2}}
\end{aligned}
$$

where $n$ is the number of Maxwell elements considered while $G_{\mathrm{i}}, \eta_{\mathrm{i}}$ and $\lambda_{\mathrm{i}}$ represent, respectively, the spring constant, the dashpot viscosity and the relaxation time of the $i^{\text {th }}$ Maxwell element. The simultaneous fitting of Equation (1) and (2) to experimental $G^{\prime}$ and $G^{\prime \prime}$ data was performed assuming that relaxation times $\left(\lambda_{\mathrm{i}}\right)$ were scaled by a factor 10 [32]. Hence, the parameters of the model are $1+n$ (i.e. $\lambda_{1}$ plus $G_{\mathrm{i}}$ ). Based on a statistical procedure [33], $n$ was selected in order to minimize the product $\chi^{2}(1+n)$, where $\chi^{2}$ is the sum of the squared errors. $G^{\prime}$ and $G^{\prime \prime}$ data represent the average of three experiments. Flory's theory (see Equation (3)) [34] enables the determination of polymeric network crosslink density $\rho_{\mathrm{x}}$ (defined as the moles of junctions between different polymeric chains per hydrogel unit volume):

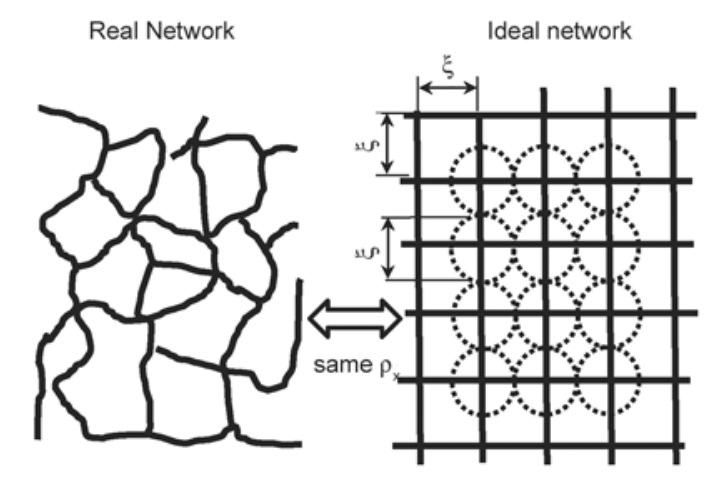

Figure 1. The equivalent network theory [36] suggests replacing the real network topology by and idealized one made up by a collection of spheres (see dotted lines) whose diameter coincides with the average real network mesh size $\xi$. The ideal network and the real one share the same crosslink density $\rho_{\mathrm{x}}$.
$\rho_{\mathrm{x}}=\frac{G}{R T}$

where $R$ is the universal gas constant, $T$ is the temperature and $G$ (shear modulus) can be computed as the sum of the elastic contributions $\left(G_{\mathrm{i}}\right)$ pertaining to each element of the generalized Maxwell model describing the hydrogel mechanical spectrum [35]. Finally, the equivalent network theory (see Equation (4)) [36] allows evaluating the average network mesh size $\xi$ (see Figure 1):

$\xi=\sqrt[3]{\frac{6}{\pi \rho_{\mathrm{x}} N_{\mathrm{A}}}}$

where $N_{\mathrm{A}}$ is the Avogadro number.

\subsection{Low field NMR characterization}

Low Field NMR (LF NMR) characterization was performed, at 25 and $37^{\circ} \mathrm{C}$, by means of a Bruker Minispec mq20 (0.47 T, $20 \mathrm{MHz}$ ). Transverse relaxation time $\left(T_{2}\right)$ measurements were carried out according to the (Carr-Purcell-Meiboom-Gill; $\mathrm{CPMG}$ ) sequence (number of scans $=4$; delay $=5 \mathrm{~s}$ ). In the case of distilled water, due to the high mobility of water molecules, the determination of the transverse relaxation time $\left(T_{2 \mathrm{H}_{2} \mathrm{O}}\right)$, at both temperatures, was determined considering three different 90-180 $0^{\circ}$ pulse separation times $(\tau): 0.25,0.5$ and $1 \mathrm{~ms}$. For each $\tau$, the time $(t)$ decay of the signal intensity $(I)$, related to the extinction of the $x-y$ component of the magnetization vector $\left(M_{\mathrm{xy}}\right)$, was fitted by the exponential function $I(t)=A_{\mathrm{H}_{2} \mathrm{O}} \mathrm{e}^{-\mathrm{t} / \mathrm{T}_{2 \mathrm{H}_{2} \mathrm{O}}}$. Thus, the water relaxation time was determined by a linear extrapolation to zero of the function $T_{2 \mathrm{H}_{2} \mathrm{O}}(\tau)\left(F_{25^{\circ} \mathrm{C}}(1,2,0.95)<642 ; F_{37^{\circ} \mathrm{C}}(1,2,0.95)\right.$ $<197)$ as suggested by the Bruker company. Accordingly, we obtained $T_{2 \mathrm{H}_{2} \mathrm{O}}^{2 \mathrm{O}^{\circ}}=3007 \pm 20 \mathrm{~ms}$ and $T_{2 \mathrm{H}_{2} \mathrm{O}}^{37^{\circ}}=$ $3694 \pm 60 \mathrm{~ms}$. In the case of hydrogels, due to the reduced water molecules mobility, there was no need to use three different $\tau$ values and the transverse relaxation time measurements were performed setting $\tau=0.5 \mathrm{~ms}$. In order to determine the $T_{2}$ discrete distribution, $I(t)$ was fitted by the following sum of exponential functions (Equation (5)):

$I(t)=\sum_{\mathrm{i}=1}^{\mathrm{m}} A_{\mathrm{i}} \mathrm{e}^{-\mathrm{t} / \mathrm{T}_{2 \mathrm{i}}} \quad\left\langle\frac{1}{T_{2}}\right\rangle=\frac{\sum_{\mathrm{i}=1}^{\mathrm{m}} \frac{A_{\mathrm{i}}}{T_{2 \mathrm{i}}}}{\sum_{\mathrm{i}=1}^{\mathrm{m}} A_{\mathrm{i}}}$

where $A_{\mathrm{i}}$ are the pre-exponential factors (dimensionless) proportional to the number of protons 
relaxing with the relaxation time $T_{2 \mathrm{i}}$ and $\left\langle 1 / T_{2}\right\rangle$ is the average value of the inverse relaxation time of protons. Again, $m$ was determined by minimizing the product $\chi^{2} \cdot(2 m)$, where $\chi^{2}$ is the sum of the squared errors and $2 m$ represents the number of fitting parameters of Equation (5) [33]. The continuous relaxation time distribution was determined according to a procedure shown in a previous work [37] (Equation (6)):

$$
\begin{aligned}
& I(t)=\int_{T_{2 \min }}^{\mathrm{T}_{2 \max }}\left(f\left(T_{2}\right) \exp \left(-\frac{t}{T_{2}}\right)\right) \mathrm{d} T_{2} \\
& f\left(T_{2}\right)=\sum_{\mathrm{i}=1}^{\mathrm{m}} \frac{B_{\mathrm{i}} \delta_{\mathrm{i}}}{\varepsilon_{\mathrm{i}}}\left(\frac{T_{2}-T_{2 \min }}{\varepsilon_{\mathrm{i}}}\right)^{\delta_{\mathrm{i}}-1} \exp \left(-\left(\frac{T_{2}-T_{2 \min }}{\varepsilon_{\mathrm{i}}}\right)^{\delta_{\mathrm{i}}}\right)
\end{aligned}
$$

$\varepsilon_{\mathrm{i}}=\left(T_{2}-T_{2 \min }\right)\left(\frac{\delta_{\mathrm{i}}}{\delta_{\mathrm{i}}-1}\right)^{1 / \delta_{\mathrm{i}}}$

where $T_{2 \max }\left(=T_{2 \mathrm{H}_{2} \mathrm{O}}\right)$ and $T_{2 \min }(=0 \mathrm{~ms})$ indicate, respectively, the lower and upper values of the continuous $T_{2}$ distribution. $B_{\mathrm{i}}, \delta_{\mathrm{i}}$ and $\varepsilon_{\mathrm{i}}$ are the $f\left(T_{2}\right)$ (sum of Weibull equations) parameters. Equation (7) simply ensures that the maximum of each Weibull distribution occurs exactly in $T_{2 \mathrm{i}}$. Equation (6) was numerically evaluated and fitted to experimental $I(t)$ data subdividing the relaxation spectrum wideness $\left(T_{2 \max }-T_{2 \min }\right)$ into 200 parts (higher subdivision was not necessary).

In order to study water mobility inside the hydrogel network, Pulsed Gradient Spin Echo (PGSE) measurements were performed. The applied sequence consists in the classical echo sequence with two equal gradient pulses (of length $\delta=0.5 \mathrm{~ms}$ ) occurring at $x_{1}=0.1 \mathrm{~ms}$ and $x_{2}=0.1 \mathrm{~ms}$ after the 90 and $180^{\circ}$ pulses, respectively. The time separation, indicated by $\Delta\left(\approx \tau-x_{1}-\delta+x_{2}\right)$, is related to the diffusion time, $t_{\mathrm{d}}$, according to $t_{\mathrm{d}}=(\Delta-\delta / 3)$. After an appropriate calibration procedure, based on the knowledge of the free water self-diffusion coefficient $\left(D_{\mathrm{H}_{2} \mathrm{O}}\right)$, it is possible measuring the average water self-diffusion coefficient inside the hydrogel $(D)$. The details of this calibration procedure can be found in the Bruker Manual, mq Gradient Unit Users Guide, version 1, January 2000, and it essentially replicates the standard procedures for the $D$ determination used in high field NMR [38]. In the case of $T_{2}$ measurements, data are the average of 27 experiments (9 repetitions for three different samples), while in the case of PGSE measurements, data are the average of 15 experiments ( 5 repetitions for three different samples).

The combination of the information coming from the relaxation and PGSE experiments were used for the estimation of the mesh size distribution of our polymeric gels. Indeed, owing to the interactions between water molecules and polymeric chains, water protons near the surface of the polymeric chains relax faster than those in the bulk $[39,40]$. On the basis of this evidence and the Scherer theory [41], we very recently demonstrated [42] that, for diluted gel systems (polymer volume fraction $\varphi \leq 0.1$ ), the average polymeric network mesh size $(\xi)$ can be expressed by Equation (8):

$\xi=\sqrt{3 \pi \frac{1-\varphi}{\varphi}} R_{\mathrm{f}}$

where $R_{\mathrm{f}}$ is the radius of the polymeric chain (see Figure 2). In addition, the 'Fiber-Cell' theory [39] ensures that the following relation holds (Equation (9)):

$$
\left\langle\frac{1}{T_{2}}\right\rangle=\frac{1}{T_{2 \mathrm{H}_{2} \mathrm{O}}}+\frac{2}{\alpha} \frac{\langle\mathcal{M}\rangle}{\xi}, \quad \alpha=\sqrt{\frac{1-\varphi}{\varphi 3 \pi}}
$$

where $\left\langle 1 / T_{2}\right\rangle$ is the inverse of the average relaxation time of the protons of the water molecules trapped within the polymeric network of the gel, $T_{2 \mathrm{H}_{2} \mathrm{O}}$ is the relaxation time of the protons of the bulk water (i.e. protons of the free water, whose relaxation is not

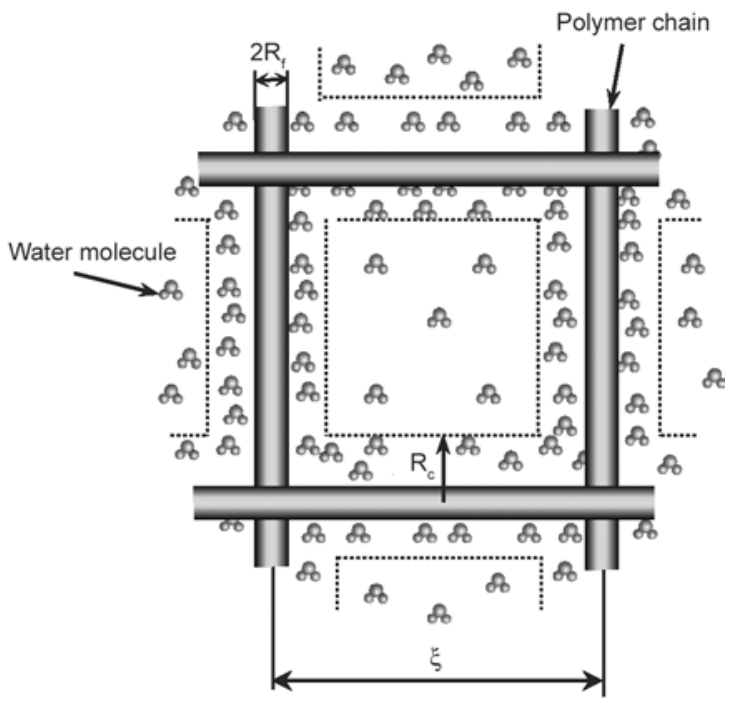

Figure 2. According to Scherer [41], the polymeric network can be represented by an ensemble of cylinders of radius $R_{\mathrm{f}}$ (fibers, i.e. polymeric chains) intersecting in a regular, cubic, array. $R_{\mathrm{c}}$ indicates the radial position where the effect of the polymeric chains on water protons relaxation becomes negligible (see dotted lines). $\xi$ is the mesh size. 
affected by the presence of the polymeric chains) and $\langle\mathcal{M}\rangle$ (length/time) is an empirical parameter, called relaxivity, accounting for the effect of polymer chains surface on proton relaxations. While Equation (9) holds on average for all the polymeric network meshes, similar expressions can be written for polymeric network meshes of different dimensions $\left(\xi_{\mathrm{i}}\right)($ Equation $(10))$ :

$$
\frac{1}{T_{2 \mathrm{i}}}=\frac{1}{T_{2 \mathrm{H}_{2} \mathrm{O}}}+\frac{2}{\alpha} \frac{\langle\mathcal{M}\rangle}{\xi_{\mathrm{i}}}
$$

where $T_{2 \mathrm{i}}$ is the relaxation time of the water molecules protons trapped in polymeric meshes of size $\xi_{\text {i }}$. Equations (9) and (10) hold in fast-diffusion regime, i.e when the mobility of the water molecules, expressed by their self-diffusion coefficient $D$, is high compared to the rate of magnetization loss, identifiable with $\langle\mathcal{M}\rangle R_{\mathrm{c}}\left(\langle\mathcal{M}\rangle R_{\mathrm{c}} / D<<1\right) . R_{\mathrm{c}}$ indicates the distance from the polymer chain axis where the effect of polymeric chains on water protons relaxation becomes negligible (see Figure 2). It can be expressed [39] by Equation (11):

$R_{\mathrm{c}}=\frac{R_{\mathrm{f}}}{\sqrt{\varphi}}$

As $\left\langle 1 / T_{2}\right\rangle$ (see Equation (5)), $T_{2 \mathrm{H}_{2} \mathrm{O}}$ and $\xi$ (see Equation (8)) are known, Equation (9) allows the determination of $\langle\mathcal{M}\rangle$. Furthermore, by knowing $\langle\mathcal{M}\rangle$ and $T_{2 \mathrm{i}}$ (see Equation (5)), Equation (10) makes possible the evaluation of $\xi_{\mathrm{i}}$ for each class of polymeric network meshes. In addition, once $\langle\mathcal{M}\rangle$ and the continuous $T_{2}$ distribution $f\left(T_{2}\right)$ (see Equation (6)) are known, it is possible determining the continuous $\xi$ distribution $f(\xi)$. Indeed, the combination of Equation (8) and (10) allows to find the relation between $\mathrm{d} \xi$ and $\mathrm{d} T_{2}$ (Equation (12)):

$$
\xi=\frac{\frac{2\langle\mathcal{M}\rangle}{\alpha}}{\frac{1}{T_{2}}-\frac{1}{T_{2 \mathrm{H}_{2} \mathrm{O}}}}, \quad \mathrm{d} \xi=\frac{2\langle\mathcal{M}\rangle}{\alpha}\left(\frac{T_{2 \mathrm{H}_{2} \mathrm{O}}}{T_{2 \mathrm{H}_{2} \mathrm{O}}-T_{2}}\right)^{2} \mathrm{~d} T_{2}
$$

Consequently, $f(\xi)$ is given by Equation (13):

$$
f(\xi)=\frac{\mathrm{d} T_{2}}{\mathrm{~d} \xi} f\left(T_{2}\right)=\frac{\sqrt{\frac{1-\varphi}{\varphi 3 \pi}}}{2\langle\mathcal{M}\rangle}\left(\frac{T_{2 \mathrm{H}_{2} \mathrm{O}}-T_{2}}{T_{2 \mathrm{H}_{2} \mathrm{O}}}\right)^{2} f\left(T_{2}\right)
$$

Furthermore, for a better comparison among different gels, it is convenient defining the probability $P(\xi)$ of finding a mesh of size $\xi$ inside the polymeric network, given by Equation (14):

$P(\xi)=\frac{f(\xi) \mathrm{d} \xi}{\int_{\xi_{\min }}^{\xi_{\max }} f(\xi) \mathrm{d} \xi}$

\subsection{Release experiments}

The hydrogels, freshly prepared in a beaker (thus in their swollen form), assumed the cylindrical shape of the vessel $($ height $=1.0 \mathrm{~cm}$, diameter $=2.2 \mathrm{~cm}$ ) and they were tested for the release of the two model drugs TPH and $\mathrm{Vit}_{12}$, at $37^{\circ} \mathrm{C}$. The gels were immersed in $200 \mathrm{~mL}\left(V_{\mathrm{r}}\right)$ of distilled water $(\mathrm{pH}=$ 5.4), and they were kept at a certain height from the bottom of the container by a thin web (see Figure 3). The medium was gently magnetically stirred and $3 \mathrm{~mL}$ samples were withdrawn from the solution at appropriate time intervals and replaced with the same amount of fresh solvent (thus, experimental concentration data were corrected for dilution). The amount of the released model drug was spectrophotometrically detected (TPH at $272 \mathrm{~nm}$, Vit. $\mathrm{B}_{12}$ at $361 \mathrm{~nm}$ ), by means of a Perkin-Elmer (lambda 3a, UV-Vis) spectrometer using quartz cells with pathlengths of 1.0 or $0.1 \mathrm{~cm}$. All experiments were car-

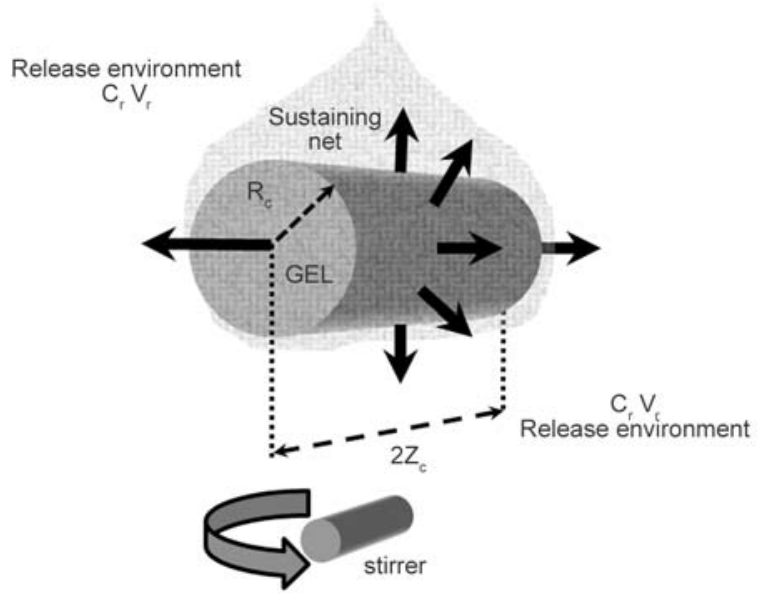

Figure 3. Drug release occurs from a cylindrical gel kept suspended in the release environment by a thin sustaining web. The release process (indicated by black thick arrows) implies the decrease of drug concentration in the gel and the contemporaneous increase of drug concentration $\left(C_{\mathrm{r}}\right)$ in the release environment (volume $V_{\mathrm{r}}$ ). Release environment homogeneity is ensured by a magnetic stirrer. 
ried out in triplicate. The possible erosion of the gel, in terms of polymer dissolution in the medium during the release experiments, was quantitatively determined by a colorimetric method [43] using phenol in the presence of sulphuric acid. Obtained results indicate that such erosion, in the first $8 \mathrm{~h}$, is almost negligible $(\leq 4 \%)$.

In order to evaluate model drug diffusion coefficients inside the gel network, release data were fitted by a mathematical model presented elsewhere [44]. Briefly, this model relays on Fick's law and on the observation that in the first 8 hours our gel did not undergo significant erosion or further swelling in the release environment. Due to gel symmetry, the intrinsically three dimensional diffusive problem could be reduced to a simpler two dimensional one (Equation (15)):

$$
\frac{\partial C}{\partial t}=\frac{D_{\mathrm{d}}}{R_{\mathrm{a}}} \frac{\partial}{\partial R_{\mathrm{a}}}\left(R \frac{\partial C}{\partial R_{\mathrm{a}}}\right)+D_{\mathrm{d}} \frac{\partial^{2} C}{\partial Z^{2}}
$$

where $D_{\mathrm{d}}$ is the drug diffusion coefficient in the gel, $t$ is time, $C$ is the drug concentration (mass/volume) in the cylinder, $R_{\mathrm{a}}$ and $Z$ are the radial and axial axes, respectively. This equation must satisfy the following initial and boundary conditions (Equations (16)-(19)):

Initial conditions:

$C\left(Z, R_{\mathrm{a}}\right)=C_{0}, \quad-Z_{\mathrm{c}} \leq Z \leq Z_{\mathrm{c}}, \quad 0 \leq R_{\mathrm{a}} \leq R_{\mathrm{c}}$

$C_{\mathrm{r}}=0$

Boundary conditions:

$C\left(Z, R_{\mathrm{c}}, t\right)=C\left( \pm Z_{\mathrm{c}}, R_{\mathrm{a}}, t\right)=k_{\mathrm{p}} \cdot C_{\mathrm{r}}(t)$

$$
V_{\mathrm{r}} C_{\mathrm{r}}(t)=\pi R_{\mathrm{c}}^{2} 2 Z_{\mathrm{c}} C_{0}-\int_{0}^{\mathrm{Z}_{\mathrm{c}}} \int_{0}^{\mathrm{R}_{\mathrm{c}}} C\left(Z, R_{\mathrm{a}}, t\right) 2 \pi R_{\mathrm{a}} \mathrm{d} R_{\mathrm{a}} \mathrm{d} Z
$$

where $2 Z_{\mathrm{c}}$ and $R_{\mathrm{c}}$ are, respectively, cylinder height and radius (see Figure 3), $C_{0}$ is the initial and uniform drug concentration in the cylinder, $C_{\mathrm{r}}$ and $V_{\mathrm{r}}$ are the drug concentration and the volume of the release medium while $k_{\mathrm{p}}$ is the drug partition coefficient between the cylindrical gel and the environmental release fluid. Equation (19) is a drug mass balance for the gel/release fluid system allowing to state the relation between $C_{\mathrm{r}}$ and $C\left(Z, R_{\mathrm{a}}, t\right)$. In order to account for the diffusion resistance exerted by the thin web surrounding the cylindrical gel (it slightly reduces release surface and favours the formation of a thick aqueous boundary layer at the gel/ release environment interface), an interfacial diffusion coefficient $\left(D_{\mathrm{di}}\right)$, lower than the bulk one $\left(D_{\mathrm{d}}\right)$, is introduced. Thus, inside the gel, drug transport is ruled by Equation (15) while, at the interface, drug transport is governed by Equation (15) where $D$ is replaced by $D_{\mathrm{di}}$. As, in our case, polymer concentration is low and model drug solubility in the release environment fluid is high, $k_{\mathrm{p}}$ can be set equal to 1 [45]. Accordingly, the proposed model is characterized by two fitting (unknowns) parameters ( $D_{\mathrm{d}}$ and $\left.D_{\mathrm{di}}\right)$. Model numerical solution was performed according to the control volume method [46] subdividing the cylindrical gel into 100 control volumes in the radial and axial directions (for a total of $10^{4}$ control volumes) and considering an integration time step equal to $22.5 \mathrm{~s}$.

Once $D_{\mathrm{d}}$ is known from Equation (15) fitting to experimental release data, the Peppas equation allows to estimate the polymeric network average mesh size $\xi$ [47] (Equation (20)):

$$
\frac{D_{\mathrm{d}}}{D_{\mathrm{d} 0}}=\left(1-\frac{2 r_{\mathrm{s}}}{\xi}\right) \exp \left(-\frac{\varphi}{1-\varphi}\right)
$$

where $r_{\mathrm{s}}$ and $D_{\mathrm{d} 0}$ indicate, respectively, the radius of the diffusing drug and its diffusion coefficient in the pure solvent filling the polymeric network.

\section{Results and discussion}

Stress sweep tests, carried out at 25 and $37^{\circ} \mathrm{C}$, indicated that for all studied systems (GG, GGb, SCLG and SCLGb), the critical deformation $\gamma_{\mathrm{c}}$ is always much higher than the constant one $(\gamma=0.01)$ applied in frequency sweep tests. Figures 4 and 5 show the mechanical spectra referring to the four systems, studied at 25 and $37^{\circ} \mathrm{C}$, respectively.

It can be noticed (see Figure 4a) that, at $25^{\circ} \mathrm{C}$, SCLG exhibits a gel behavior as the elastic modulus $\left(G^{\prime}\right)$ is always bigger than the viscous one $\left(G^{\prime \prime}\right)$ and both are, almost, pulsation $(\omega)$ independent. On the other side, GG shows the typical solution behavior as $G^{\prime \prime}$ prevails on $G^{\prime}$ and both moduli depend on $\omega$. Five and four Maxwell elements are, respectively, necessary for a statistically good description (see $F$ test values in Figure 4a caption) of the GG and SCLG mechanical spectra (see fitting parameters values in Table 1). Figure $4 \mathrm{~b}$ clearly shows the different effect of borax addition to the GG and SCLG systems. In fact, while borax presence plays a marginal role in 

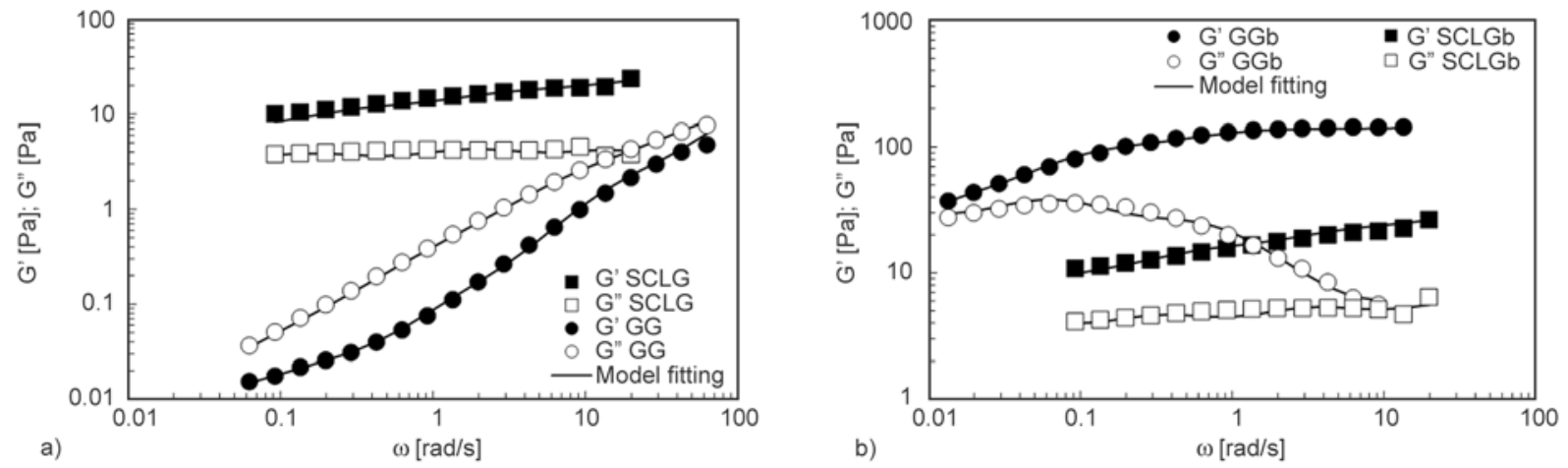

Figure 4. a) mechanical spectra ( $G^{\prime}$ elastic modulus, $G^{\prime \prime}$ viscous modulus) referring to the Guar gum $(\mathrm{GG})$ and Scleroglucan (SCLG) systems at $25^{\circ} \mathrm{C}$. Solid lines indicate the best fitting of the generalized Maxwell Model (Equations (1), (2)) ( $F$ test always positive: $\left.F_{\mathrm{GG}}(5,32,0.95)<150, F_{\mathrm{SCLG}}(4,25,0.95)<374\right)$. b) mechanical spectra $\left(G^{\prime}\right.$ elastic modulus, $G^{\prime \prime}$ viscous modulus) referring to the Guar gum/borax (GGb) and Scleroglucan/borax (SCLGb) systems at $25^{\circ} \mathrm{C}$. Solid lines indicate the best fitting of the generalized Maxwell Model (Equations (1), (2)) ( $F$ test always positive: $\left.F_{\mathrm{GGb}}(4,32,0.95)<103.5, F_{\mathrm{SCLGb}}(5,24,0.95)<251\right)$.

Table 1. Generalized Maxwell model (Equations (1), (2)) parameters ( \pm standard deviation) deriving from the fitting of data shown in Figures 1 and 2. $\lambda_{1}$ is the first relaxation time, $G_{\mathrm{i}}$ represents the spring constant of the ith Maxwell element, $G$ is the shear modulus (sum of all $G_{\mathrm{i}}$ ), $\rho_{\mathrm{x}}$ is the crosslink density calculated according to Equation (3), while $\xi$ is the average network mesh size calculated according to Equation (4).

\begin{tabular}{|c|c|c|c|c|}
\hline $25^{\circ} \mathrm{C}$ & GG & SCLG & GGb & SCLGb \\
\hline$\lambda_{1}[\mathrm{~s}]$ & $(7.7 \pm 3) \cdot 10^{-3}$ & $(81.7 \pm 11) \cdot 10^{-3}$ & $(15.7 \pm 4) \cdot 10^{-3}$ & $(30.0 \pm 5) \cdot 10^{-3}$ \\
\hline$G_{1}[\mathrm{~Pa}]$ & $20.3 \pm 4.4$ & $7.0 \pm 0.4$ & $21.8 \pm 6.1$ & $9.7 \pm 0.84$ \\
\hline$G_{2}[\mathrm{~Pa}]$ & $2.3 \pm 1.1$ & $6.2 \pm 0.36$ & 0 & $7.4 \pm 0.5$ \\
\hline$G_{3}[\mathrm{~Pa}]$ & $0.12 \pm 0.07$ & $5.0 \pm 0.37$ & $36.5 \pm 8.4$ & $6.4 \pm 0.5$ \\
\hline$G_{4}[\mathrm{~Pa}]$ & $0.014 \pm 0.007$ & $7.4 \pm 0.53$ & $61.6 \pm 9.8$ & $6.1 \pm 0.76$ \\
\hline$G_{5}[\mathrm{~Pa}]$ & $0.012 \pm 0.004$ & - & $42.0 \pm 13$ & $4.0 \pm 0.9$ \\
\hline$G[\mathrm{~Pa}]$ & $22.9 \pm 4.5$ & $25.6 \pm 0.8$ & $162 \pm 19$ & $33.6 \pm 1.6$ \\
\hline$\rho_{\mathrm{x}}\left[\mathrm{mol} / \mathrm{cm}^{3}\right]$ & - & $(1.0 \pm 0.03) \cdot 10^{-8}$ & $(6.5 \pm 0.79) \cdot 10^{-8}$ & $(1.4 \pm 0.06) \cdot 10^{-8}$ \\
\hline$\xi[\mathrm{nm}]$ & - & $67.4 \pm 0.8$ & $36.5 \pm 1.4$ & $61.6 \pm 1.0$ \\
\hline $37^{\circ} \mathrm{C}$ & GG & SCLG & GGb & SCLGb \\
\hline$\lambda_{1}[\mathrm{~s}]$ & $(11.6 \pm 2) \cdot 10^{-3}$ & $(62.5 \pm 6.6) \cdot 10^{-3}$ & $(22.7 \pm 6.4) \cdot 10^{-3}$ & $(18.9 \pm 2) \cdot 10^{-3}$ \\
\hline$G_{1}[\mathrm{~Pa}]$ & $7.6 \pm 1.1$ & $5.4 \pm 0.23$ & $7.9 \pm 2.5$ & $8.3 \pm 0.32$ \\
\hline$G_{2}[\mathrm{~Pa}]$ & $0.40 \pm 0.22$ & $4.1 \pm 0.16$ & $10.0 \pm 3.8$ & $5.5 \pm 0.27$ \\
\hline$G_{3}[\mathrm{~Pa}]$ & $0.049 \pm 0.045$ & $2.9 \pm 0.15$ & $36.1 \pm 3.8$ & $4.0 \pm 0.2$ \\
\hline$G_{4}[\mathrm{~Pa}]$ & $0.04 \pm 0.038$ & 0 & $9.8 \pm 2.9$ & $3.5 \pm 0.24$ \\
\hline$G_{5}[\mathrm{~Pa}]$ & $0.069 \pm 0.043$ & $1.8 \pm 0.5$ & $1.6 \pm 0.6$ & $1.5 \pm 0.3$ \\
\hline$G[\mathrm{~Pa}]$ & $8.2 \pm 1.1$ & $14.2 \pm 0.75$ & $65.5 \pm 6.6$ & $22.9 \pm 0.6$ \\
\hline$\rho_{\mathrm{x}}\left[\mathrm{mol} / \mathrm{cm}^{3}\right]$ & - & $(5.5 \pm 0.3) \cdot 10^{-9}$ & $(2.5 \pm 0.25) \cdot 10^{-8}$ & $(8.9 \pm 0.2) \cdot 10^{-9}$ \\
\hline$\xi[\mathrm{nm}]$ & - & $83.2 \pm 1.5$ & $49.9 \pm 1.7$ & $71.0 \pm 0.6$ \\
\hline
\end{tabular}

the case of SCLG (only a very small increase of the moduli can be noticed), for the GG system a relevant change occurs in the mechanical properties. Now, $G^{\prime}$ prevails over $G^{\prime \prime}$ in the whole experimental frequency window and the crossing point is around $\omega=0.01 \mathrm{rad} / \mathrm{s}$. The $\omega$ dependence of $G^{\prime}$ and $G^{\prime \prime}$ witnesses the weak nature of the formed hydrogel. Also in this case, the generalized Maxwell model provides a statistically good fitting (see $F$ test values in Figure 4b caption) adopting, respectively, four and five elements for the GGb and SCLGb systems.

Temperature increase to $37^{\circ} \mathrm{C}$ does not modify the gel and the solution nature of the SCLG and GG systems. Nevertheless $G^{\prime}$ and $G^{\prime \prime}$, for both systems, are reduced (see Figure 5a). Again, borax addition (Figure 5b) implies a moderate increase of the SCLGb moduli and a considerable modification of the GG characteristics. Indeed, not only $G^{\prime}$ and $G^{\prime \prime}$ are clearly increased (about one order of magnitude) but also 

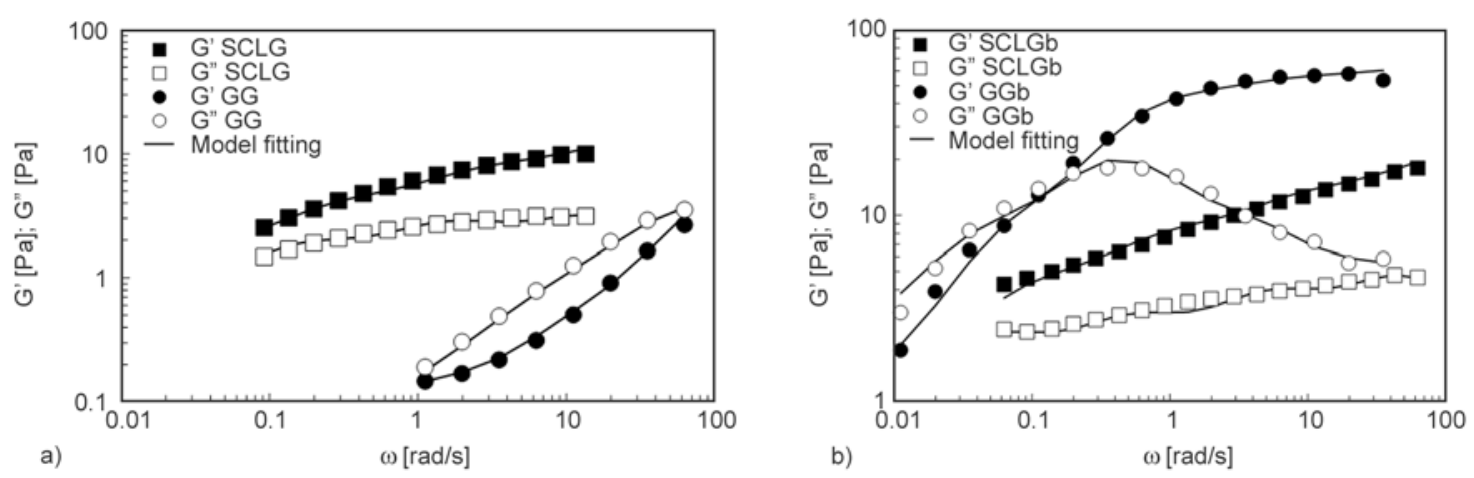

Figure 5. a) mechanical spectra ( $G^{\prime}$ elastic modulus, $G^{\prime \prime}$ viscous modulus) referring to the Guar gum $(\mathrm{GG})$ and Scleroglucan (SCLG) systems at $37^{\circ} \mathrm{C}$. Solid lines indicate the best fitting of the generalized Maxwell Model (Equations (1), (2)) $\left(F\right.$ test always positive: $\left.F_{\mathrm{GG}}(5,10,0.95)<38.1, F_{\mathrm{SCLG}}(5,22,0.95)<504.5\right)$. b) mechanical spectra $\left(G^{\prime}\right.$ elastic modulus, $G^{\prime \prime}$ viscous modulus) referring to the Guar gum/borax (GGb) and Scleroglucan/borax (SCLGb) systems at $37^{\circ} \mathrm{C}$. Solid lines indicate the best fitting of the generalized Maxwell Model (Equations (1), (2)) ( $F$ test always positive: $\left.F_{\mathrm{GGb}}(5,24,0.95)<55.4, F_{\mathrm{SCLGb}}(5,32,0.95)<2076\right)$.

the system behavior shifts from that of a solution (GG) to that of an incipient weak gel (GGb) as testified by the presence of the crossover point (detectable at $\omega \approx 0.2 \mathrm{rad} / \mathrm{s}$ ) where system elastic and viscous characteristics are equal. This means that the system is moving from a sol to a gel condition, i.e, it lies in a sort of transition zone. Generalized Maxwell model gives a statistically good fitting of data shown in Figure 5a and $5 \mathrm{~b}$ (see $F$ test values in the captions to this figures) assuming four or five Maxwell elements (see Table 1). In conclusion, rheological characterization evidences the marked effect of borax addition to the GG system and the weak effect exerted on the SCLG system at both temperatures. While SCLG always exhibits gel properties, GG system becomes a weak gel (or incipient weak gel) only in the presence of borax.

On the basis of the crosslink density $\left(\rho_{\mathrm{x}}\right)$ evaluation (see Equation (3)), equivalent network theory (see Equation (4)) allows to estimate, for the gel systems, the average network mesh size $\xi$. Table 1 shows that $\xi$ spans from $36.5 \mathrm{~nm}\left(\mathrm{GGb}, 25^{\circ} \mathrm{C}\right)$ to $83.2 \mathrm{~nm}\left(\mathrm{SCLG}, 37^{\circ} \mathrm{C}\right)$. The high $\xi$ values witness the low connectivity of all tested gels.

LF NMR analysis indicates that, regardless of temperature, GG is characterized by only one relaxation time $\left(T_{21}\right)$ (see Table 2), as expected for aqueous solutions, while the relaxation of the protons belonging to the polymeric chains are not detectable, due to their very low amount (around $0.5 \%$ ) in comparison with those of water. Thus, LF NMR and rheology analysis lead to the same conclusion: the GG system is an aqueous polymer solution at both temperatures.
Table 2. Relaxation times, $T_{22}$ and $T_{21} \pm$ standard deviation, and weight [\%], $A_{1} \%$ and $A_{2} \% \pm$ standard deviation, referring to GG, SCLG, GGb, SCLGb and distilled water, at 25 and $37^{\circ} \mathrm{C} . \quad A_{\mathrm{i}} \%=$ $100 \cdot A_{\mathrm{i}} /\left(A_{1}+A_{2}\right)$

\begin{tabular}{|c|c|c|c|c|c|}
\hline $\mathrm{T}\left[{ }^{\circ} \mathrm{C}\right]$ & & $\mathrm{T}_{21}[\mathrm{~ms}]$ & $\mathrm{A}_{1} \%$ & $\mathrm{~T}_{22}[\mathrm{~ms}]$ & $\mathrm{A}_{2} \%$ \\
\hline \multirow{5}{*}{25} & GG & $2032 \pm 92$ & 100 & & \\
\hline & GGb & $1527 \pm 46$ & 100 & & \\
\hline & SCLG & $1359 \pm 23$ & 100 & & \\
\hline & SCLGb & $343 \pm 84$ & $84.5 \pm 23.5$ & $173 \pm 77$ & $15.5 \pm 7.8$ \\
\hline & water & $3007 \pm 20$ & 100 & & \\
\hline & & & & & \\
\hline \multirow{5}{*}{37} & GG & $2466 \pm 80$ & 100 & & \\
\hline & GGb & $1912 \pm 94$ & 100 & & \\
\hline & SCLG & $1321 \pm 46$ & 100 & & \\
\hline & SCLGb & $451 \pm 74$ & 100 & - & - \\
\hline & water & $3694 \pm 60$ & 100 & & \\
\hline
\end{tabular}

The addition of borax to the GG system induces a significant reduction of $T_{21}$ at both temperatures. Since the addition of borax to distilled water does not substantially modify the water relaxation time $T_{2 \mathrm{H}_{2} \mathrm{O}}$ (data not shown), we can assert that the observed reduction of $T_{21}$, at both temperatures, cannot directly depend on the presence of borax. Thus, the $T_{21}$ reduction is in agreement with the formation of a new, more compact, architecture of the polymeric chains (gel network) related to the addition of borax. It is interesting to point out that, in the case of a dextran system $\left.\left(c_{\mathrm{p}}=0.7 \% \mathrm{w} / \mathrm{v}\right)\right)$, the addition of borax $(r=$ borax moles $/$ dextran moles $=1$; data not shown) does not modify $T_{21}$, this being a clear indication that, in this case, the presence of borax does not lead to the formation of a polymeric network. It is also interesting to notice that the relative increase of $T_{21}$ with temperature is similar for both, GGb 
$(25 \%)$ and GG (21\%) systems (see Table 2). This result shows that the interactions among polymeric chains and water molecules are not so strong, as expected for a weak gel.

Although the SCLG system shows, as GG, only one relaxation time at 25 and $37^{\circ} \mathrm{C}$, it cannot be considered a solution, because $T_{21}$ is, essentially, temperature independent and this behavior should not occur in a polymeric solution at such low concentration $\left(c_{\mathrm{p}}=0.7 \% \mathrm{v} / \mathrm{w}\right)$. In addition, also the relatively small $T_{21}$ value (recorded at both temperatures) with respect to that of water, $T_{2 \mathrm{H}_{2} \mathrm{O}}$, is not compatible with such a low $c_{\mathrm{p}}$. Finally, Table 2 shows that the addition of borax to the SCLG system implies a drastic reduction of the average relaxation time $\left\langle T_{2}\right\rangle$ at $25^{\circ} \mathrm{C}(317 \mathrm{~ms})$ and $37^{\circ} \mathrm{C}(451 \mathrm{~ms})$. Again, the essential $\left\langle T_{2}\right\rangle$ independence on temperature and its high reduction in comparison with water, $\left\langle T_{2 \mathrm{H}_{2} \mathrm{O}}\right\rangle$, indicates the gel nature of the SCLGb system. The fact that, for this last system, two relaxation times were detected at $25^{\circ} \mathrm{C}$ and only one at $37^{\circ} \mathrm{C}$ can be explained by the formation, at $37^{\circ} \mathrm{C}$, of a more homogeneous polymeric network due to a higher mobility of borax ions in the initial polymer solution. In conclusion, as far as the macroscopic behavior is concerned, the relaxation analysis shows the same findings obtained from the rheological characterization of our polymeric systems. On the other side, some differences arose in the estimation of $\xi$. Indeed, knowing that $\varphi=4.35 \cdot 10^{-3}$ and $R_{\mathrm{f}} \approx 2.15 \mathrm{~nm}$ for both GG and SCLG systems [48], the application of Equation (8) leads to $\xi=99.8 \mathrm{~nm}$. The comparison between the values reported in Table 1 ( 25 and $37^{\circ} \mathrm{C}$ ) and Table 3 shows that the $\xi$ values from rheological approach are about $40 \%$ for $\mathrm{GGb}$ and about $75 \%$ for SCLG and SCLGb of those obtained by LF NMR theory. Nevertheless, taking into account the simplifications adopted in both approaches, we believe that these cannot be considered as significant differences.

In order to estimate the continuous mesh size distribution of GGb, SCLG and SCLGb systems, Equation (9) was used for the determination of parameter $\langle\mathcal{M}\rangle$ whose values are reported in Table 3 . It can be seen that $\langle\mathcal{M}\rangle$ values, regardless of temperature, increase going from GGb to SCLG and SCLGb systems. This indicates a stronger and stronger effect of polymer chain surfaces on the relaxation of protons. The diffusion coefficient, $D$, was practically constant with the diffusion time $t_{\mathrm{d}}$ for all studied systems (see, Figure 6), and therefore the check on the fast diffusion conditions (evaluation of the dimensionless parameter $\langle\mathcal{M}\rangle R_{\mathrm{c}} / D$ ) was carried out taking into account the $D$ value averaged on the measurements performed at all $t_{\mathrm{d}}$.

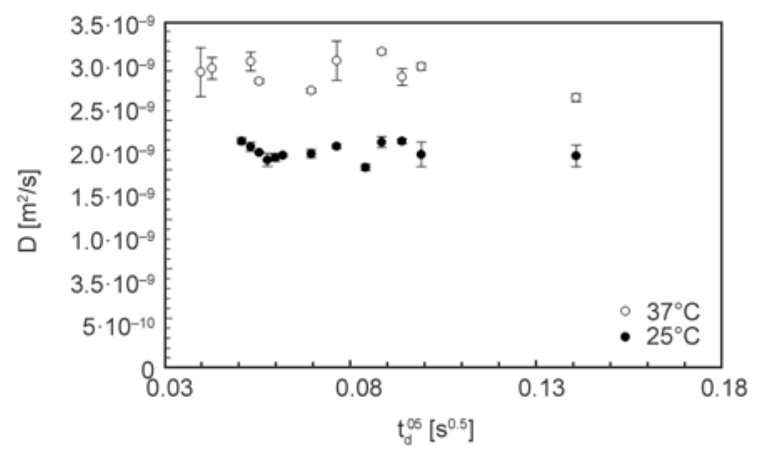

Figure 6. Experimental dependence of the water self-diffusion coefficient $(D)$ on the square root of the diffusion time $t_{\mathrm{d}}$ for the Guar gum/borax gel (GGb) at 25 and $37^{\circ} \mathrm{C}$. For $t_{\mathrm{d}}=0$, water self-diffusion coefficient at 25 and $37^{\circ} \mathrm{C}$ is, respectively, $2.3 \cdot 10^{-9}$ and $3.04 \cdot 10^{-9} \mathrm{~m}^{2} / \mathrm{s}[50]$. Vertical bars indicate standard error.

Table 3. Average relaxation time $\left\langle T_{2}\right\rangle \pm$ standard deviation, water self-diffusion coefficient $D \pm$ standard deviation, average effect of surface on protons relaxation $\langle\mathcal{M}\rangle \pm$ standard deviation, $R_{\mathrm{c}}=32.6 \mathrm{~nm}$ (see Equation (11)) and mesh diam-

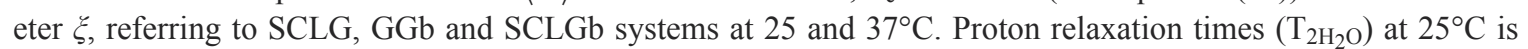
$3007 \pm 20 \mathrm{~ms}$ while at $37^{\circ} \mathrm{C}$ it is $3694 \pm 60 \mathrm{~ms}$.

\begin{tabular}{|c|c|c|c|c|c|c|}
\hline $\mathrm{T}\left[{ }^{\circ} \mathbf{C}\right]$ & & $\begin{array}{l}\left\langle\mathrm{T}_{2}\right\rangle \\
{[\mathrm{ms}]}\end{array}$ & $\begin{array}{c}\xi \\
{[\mathrm{nm}]}\end{array}$ & $\begin{array}{l}\mathrm{D} \cdot 10^{9} \\
{\left[\mathrm{~m}^{2} / \mathrm{s}\right]}\end{array}$ & $\begin{array}{l}\langle\mathcal{M}\rangle \cdot 10^{3} \\
{[\mathrm{~nm} / \mathrm{ms}]}\end{array}$ & $\frac{\langle\mathscr{M}\rangle \mathbf{R}_{\mathbf{c}}}{\mathbf{D}} \cdot 10^{6}$ \\
\hline \multirow{3}{*}{$25^{\circ} \mathrm{C}$} & GGb & $1527 \pm 46$ & 99.8 & $2.2 \pm 0.1$ & $62 \pm 6$ & 1.2 \\
\hline & SCLG & $1359 \pm 23$ & 99.8 & $2.2 \pm 0.1$ & $99 \pm 3$ & 1.5 \\
\hline & SCLGb & $317 \pm 23$ & $\begin{array}{c}109 \pm 16(84.5 \%) \\
31 \pm 9(15.5 \%)\end{array}$ & $2.3 \pm 0.1$ & $693 \pm 263$ & 10.0 \\
\hline \multirow{3}{*}{$37^{\circ} \mathrm{C}$} & GGb & $1912 \pm 94$ & 99.8 & $2.9 \pm 0.2$ & $79 \pm 5$ & 0.7 \\
\hline & SCLG & $1321 \pm 46$ & 99.8 & $2.8 \pm 0.2$ & $119 \pm 6$ & 1.4 \\
\hline & SCLGb & $451 \pm 74$ & 99.8 & $2.9 \pm 0.2$ & $477 \pm 89$ & 5.4 \\
\hline
\end{tabular}




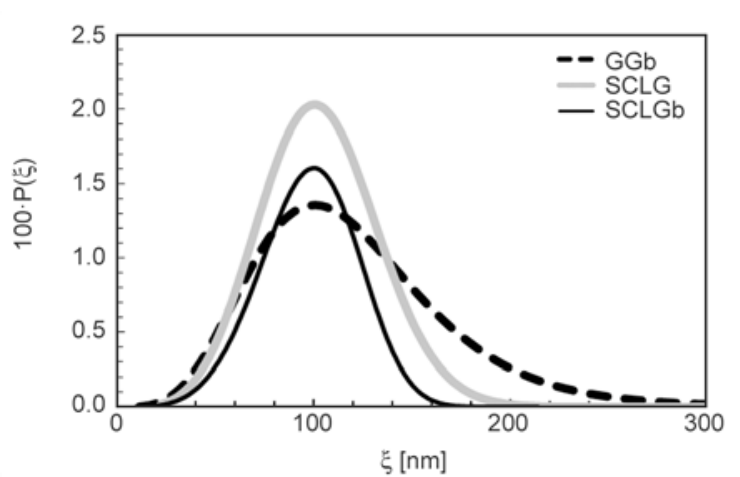

Figure 7. Mesh size distribution, $P(\xi)$, of GGb (Guar gum/ borax), SCLG (Scleroglucan) and SCLGb (Scleroglucan/borax) hydrogels at $37^{\circ} \mathrm{C}$

Table 3 clearly shows that in all gel systems, $\langle\mathcal{M}\rangle R_{\mathrm{C}} / D$ was much less than 1, i.e. fast diffusion conditions always apply. Accordingly, Equation (10) and (13) can be used for the estimation of $f\left(T_{2}\right), f(\xi)$ and, finally of $P(\xi)$, as shown in Figure $7\left(37^{\circ} \mathrm{C}\right)$.

Figure 7 shows that, for GGb, the mesh size distribution spans, approximately, from 10 to $300 \mathrm{~nm}$ while it is slightly less wide for SCLG and SCLGb. As the $\xi$ values estimated by means of rheology experiments fall inside these distributions and they are not too far from the distribution peaks, we may conclude that the two approaches do not lead to very different results (see Table 1).

Another approach to acquire some insight about polymeric network characteristics is the determination of model molecule diffusion coefficients. For this purpose, two model drugs, TPH and Vit $\mathrm{B}_{12}$, were considered. Figure 8 reports TPH and Vit $\mathrm{B}_{12}$ release from $\mathrm{GGb}$ at $37^{\circ} \mathrm{C}$. These two release kinetics are fitted by means of Equation (15) knowing that the release volume $V_{\mathrm{r}}=200 \mathrm{~cm}^{3}$ and the initial drug concentration $C_{0}$ in the gel is equal to $5.2 \mathrm{mg} / \mathrm{cm}^{3}$ for the two drugs. In both cases, the fitting is statistically good as proved by the $F$ test $\left(F_{\mathrm{TPH}}(1,10,0.95)\right.$ $\left.<75 ; F_{\mathrm{Vit}_{12}}(1,10,0.95)<30627\right)$. In the case of TPH we have $D_{\mathrm{d}}=D_{\mathrm{di}}=(2.7 \pm 0.5) \cdot 10^{-10} \mathrm{~m}^{2} / \mathrm{s}$ while, for Vit $\mathrm{B}_{12}$, model fitting yields to $D_{\mathrm{d}}=$ $(2.3 \pm 0.1) \cdot 10^{-10} \mathrm{~m}^{2} / \mathrm{s}$ and $D_{\mathrm{di}}=(3.0 \pm 0.1) \cdot 10^{-11} \mathrm{~m}^{2} / \mathrm{s}$. These results indicate that the resistance due to the thin web, suspending the gel in the release environment, is negligible for TPH while it plays a significant role in the case of Vit $\mathrm{B}_{12}$. This seems reasonable if we consider the different model drug dimensions (TPH van der Waals radius $r=3.7 \AA$; Vit $\mathrm{B}_{12}$ van der Waals radius $r=8.5 \AA$ ) [45]. On the basis of the diffusion coefficients in water at $37^{\circ} \mathrm{C}$ of TPH $\left(D_{\mathrm{d} 0}=8.2 \cdot 10^{-10} \mathrm{~m}^{2} / \mathrm{s}\right)$ and of Vit B $12\left(D_{\mathrm{d} 0}=\right.$

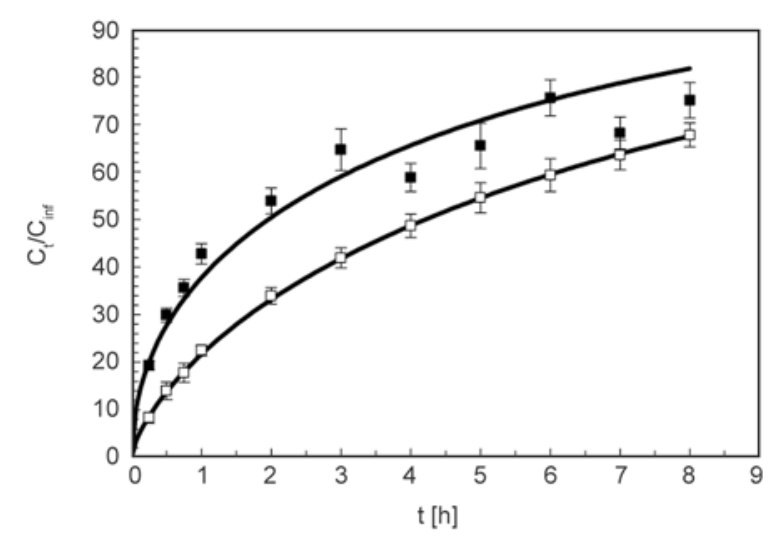

Figure 8. Theophylline (TPH; filled squares) and Vit $\mathrm{B}_{12}$ release (open squares) from $\mathrm{GGb}$ hydrogel at $37^{\circ} \mathrm{C}$ (vertical bars indicate standard error). $C_{\mathrm{t}}$ and $C_{\text {inf }}$ are, respectively, drug concentration at time $t$ and after an infinite time. Solid lines indicate model best fitting (Equation (15)).

$3.8 \cdot 10^{-10} \mathrm{~m}^{2} / \mathrm{s}$ ) [45], Equation (20) allows estimating the polymeric network mesh size $(\xi)$ knowing that polymer volume fraction $(\varphi)$ in GGb hydrogel is equal to $4.35 \cdot 10^{-3}$, it turns out that $\xi=(1.1 \pm 0.1)$ and (4.3 \pm 0.06$) \mathrm{nm}$ in the case of TPH and Vit $\mathrm{B}_{12}$, respectively. These values are much smaller than those estimated by means of rheology and LF NMR (49.9 nm, see Table 1, and $99.8 \mathrm{~nm}$, see Table 3). Due to the low polymer concentration and crosslink density of GGb hydrogel, the $\xi$ values estimated according to Equation (20) seem too small. The motivation for the Equation (20) failure should rely on the fact that $\mathrm{GGb}$ system, at $37^{\circ} \mathrm{C}$, represents an incipient hydrogel condition (as previously discussed, see also Figure 5b). Here, drug diffusion is not only affected by the presence of the structured polymeric network but also by the chains that, although bound to the network to one end, can freely fluctuate among meshes. These chains are elastically inactive but can hinder drug diffusion through a viscous drag, favoured by weak van der Walls interactions with drug molecules. Tomic and coworkers [49] found that also very weak van der Waals interactions are sufficient to hinder drug molecule movements in the presence of a polymeric network with mesh size much larger than the drug molecular size. In addition, the rheological analysis suggests that the number of elastically inactive chains is high, as proved by the importance of the viscous contribution to the GGb mechanical behavior (see Figure 5b). On the contrary, in the case of a true hydrogel, such as SCLGb (where the viscous contribution is low), we found a good agreement 
between the estimation of $\xi$ according to the rheological approach and Equation (20) [44].

\section{Conclusions}

The rheological and LF NMR analyses allow concluding that, regardless of temperature, the GG system $\left(c_{\mathrm{p}}=0.7 \%\right)$ behaves as a polymeric solution while SCLG $\left(c_{\mathrm{p}}=0.7 \%\right)$ is a gel. The addition of borax to $\mathrm{GG}\left(c_{\mathrm{p}}=0.7 \%\right)$ appreciably modifies the system behavior leading to the formation of a weak hydrogel that, at $37^{\circ} \mathrm{C}$, can be considered as an incipient weak gel. The evaluation of the polymeric network mesh size by rheology resulted to be slightly smaller than that derived from the LF NMR approach (mesh size distribution spanning from 10 to $300 \mathrm{~nm}$ ). On the other side, the addition of borax to SCLG mainly appears at the nanoscale level. In fact, while gel strength is slightly increased, borax leads to a considerable reduction of the proton average relaxation times. The high values of the average polymeric network mesh size of all the studied gels (GGb, SCLG, SCLGb) indicate their low connectivity. In all tested gels (GGb, SCLG and SCLGb), the fast condition diffusion was verified. In the case of GGb hydrogel, rheology and LF NMR led to an estimations of the average mesh size that does not agree with that calculated from the model drug diffusion coefficient inside the gel. As the rheology - LF NMR estimations seems to be the correct ones, the failure of the diffusion coefficient approach should rely on the nature of the GGb polymeric network. Actually, in a hydrogel where the viscous component is high, model drug movement is not only reduced by the presence of the polymeric network (elastically active chains) but also by elastic inactive chains that, although bound to the network, can freely fluctuate and can exert a viscous drag, favored by weak van der Waals interactions, on the moving model drug molecules. This hypothesis is supported by the fact that, in the case of SCLGb, where the viscous component is not so important, rheology approach and the diffusion coefficient approach lead to a similar estimation of the average mesh size.

\section{Acknowledgements}

Financial support from Sapienza University of Rome 'Ricerche Universitarie' 2011, n. C26A119N2S and 'Fondo PRIN 2010-2011 (20109PLMH2)', are acknowledged.

\section{References}

[1] Maier H., Anderson M., Karl C., Magnuson K., Whistler R. L.: Guar, locust bean gum, tara, and fenugreek gums. in 'Industrial gums: polysaccharides and their derivatives' (eds.: Whistler R. L., BeMiller J. N.) Academic Press, New York, 181-226 (1993).

[2] McCleary B. V., Clark A. H., Dea I. C. M., Rees D. A.: The fine structures of carob and guar galactomannans. Carbohydrate Research, 139, 237-260 (1985).

DOI: $10.1016 / 0008-6215(85) 90024-2$

[3] Fox J. E.: Seed gums. in 'Thickening and gelling agents for food' (ed.: Imeson A.) Blackie Academic Professional, New York, 262-283 (1997).

[4] Kucera C. H., DeMott D. N.: Drilling fluid containing crosslinked polysaccharide derivative. U.S. patent 4257903, USA (1981).

[5] Brode G. L., Goddard E. D., Harris W. C., Salensky G. A.: Cationic polysaccharides for cosmetics and therapeutics. in 'Cosmetic and pharmaceutical applications of polymers' (eds.: Gebelein C. G., Cheng T. C., Yang V. C.) Plenum Press, New York, 117-128 (1991).

[6] Vijayendran B. R., Bone T.: Absolute molecular weight and molecular weight distribution of guar by size exclusion chromatography and low-angle laser light scattering. Carbohydrate Polymers, 4, 299-313 (1984).

DOI: 10.1016/0144-8617(84)90005-5

[7] Picout D. R., Ross-Murphy S. B.: On the MarkHouwink parameters for galactomannans. Carbohydrate Polymers, 70, 145-148 (2007).

DOI: $10.1016 /$ j.carbpol.2007.03.010

[8] Gliko-Kabir I., Yagen B., Penasi A., Rubinstein A.: Low swelling, crosslinked guar and its potential use as colonspecific drug carrier. Pharmaceutical Research, 15, 1019-1025 (1998). DOI: 10.1023/A:1011921925745

[9] Coviello T., Alhaique F., Dorigo A., Matricardi P., Grassi M.: Two galactomannans and scleroglucan as matrices for drug delivery: Preparation and release studies. European Journal of Pharmaceutics and Biopharmaceutics, 66, 200-209 (2007).

DOI: 10.1016/j.ejpb.2006.10.024

[10] Norisuye T., Yanaki T., Fujita H.: Triple helix of a Schizophyllum commune polysaccharide in aqueous solution. Journal of Polymer Science: Polymer Physics Edition, 18, 547-558 (1980). DOI: $10.1002 /$ pol.1980.180180314

[11] Yanaki T., Norisuye T., Fujita H.: Triple helix of Schizophyllum commune polysaccharide in dilute solution. 3 . Hydrodynamic properties in water. Macromolecules, 13, 1462-1466 (1980). DOI: $10.1021 / \mathrm{ma} 60078 \mathrm{a} 019$

[12] Giavasis I., Harvey L. M., McNeil B.: Scleroglucan. in 'Biopolymers, polysaccharides II' (eds.: De Baets S., Vandamme E. J., Steinbüchel A.) Wiley, Weinheim, 37-60 (2002). 
[13] Rizk S., Duru C., Gaudy D., Jacob M., Ferrari F., Bertoni M., Caramella C.: Physico-chemical characterization and tabletting properties of Scleroglucan. International Journal of Pharmaceutics, 112, 125-131 (1994). DOI: $10.1016 / 0378-5173(94) 90422-7$

[14] Pezron E., Leibler L., Lafuma F.: Complex formation in polymer-ion solutions. 2. Polyelectrolyte effects. Macromolecules, 22, 2656-2662 (1989).

DOI: $10.1021 / \mathrm{ma} 00196 \mathrm{a} 021$

[15] Shibayama M., Hiroyuki Y., Hidenobu K., Hiroshi F., Shunji N.: Sol-gel transition of poly(vinyl alcohol)borate complex. Polymer, 29, 2066-2071 (1988).

DOI: 10.1016/0032-3861(88)90182-6

[16] Bocchinfuso G., Palleschi A., Mazzuca C., Coviello T., Alhaique F., Marletta G.: Theoretical and experimental study on a self-assembling polysaccharide forming nanochannels: Static and dynamic effects induced by a soft confinement. The Journal of Physical Chemistry B, 112, 6473-6483 (2008).

DOI: $10.1021 / \mathrm{jp} 076074 \mathrm{f}$

[17] Palleschi A., Coviello T., Bocchinfuso G., Alhaique F.: Investigation on a new scleroglucan/borax hydrogel: Structure and drug release. International Journal of Pharmaceutics, 322, 13-21 (2006).

DOI: 10.1016/j.ijpharm.2006.05.029

[18] Kesavan S., Prud'homme R. K.: Rheology of guar and (hydroxypropyl) guar crosslinked by borate. Macromolecules, 25, 2026-2032 (1992).

DOI: $10.1021 / \mathrm{ma} 00033 \mathrm{a} 029$

[19] Tayal A., Pai V. B., Khan S. A.: Rheology and microstructural changes during enzymatic degradation of a guar-borax hydrogel. Macromolecules, 32, 55675574 (1999).

DOI: $10.1021 / \mathrm{ma} 990167 \mathrm{~g}$

[20] Pezron E., Leibler L., Ricard A., Audebert R.: Reversible gel formation induced by ion complexation. 2. Phase diagrams. Macromolecules, 21, 1126-1131 (1988).

DOI: $10.1021 / \mathrm{ma} 00182 \mathrm{a} 046$

[21] Pezron E., Ricard A., Lafuma F., Audebert R.: Reversible gel formation induced by ion complexation. 1. Borax-galactomannan interactions. Macromolecules, 21, 1121-1125 (1988).

DOI: $10.1021 / \mathrm{ma} 00182 \mathrm{a} 045$

[22] Pezron E., Leibler L., Ricard A., Lafuma F., Audebert R.: Complex formation in polymer-ion solution. 1. Polymer concentration effects. Macromolecules, 22, 1169-1174 (1989).

DOI: $10.1021 / \mathrm{ma} 00193 \mathrm{a} 030$

[23] Fang Y., Takahashi R., Nishinari K.: A gel network constituted by rigid schizophyllan chains and nonpermanent cross-links. Biomacromolecules, 5, 126-136 (2004).

DOI: $10.1021 / \mathrm{bm} 034261 \mathrm{n}$

[24] Grisel M., Muller G.: Rheological properties of the schizophyllan-borax system. Macromolecules, 31, 4277-4281 (1998).

DOI: $\underline{10.1021 / \mathrm{ma} 970485 \mathrm{k}}$
[25] Bluhm L. T., Deslandes Y., Marchessault R. H., Pérez S., Rinaudo M.: Solid-state and solution conformation of scleroglucan. Carbohydrate Research, 100, 117130 (1982). DOI: 10.1016/S0008-6215(00)81030-7

[26] Sandolo C., Matricardi P., Alhaique F., Coviello T.: Effect of temperature and cross-linking density on rheology of chemical cross-linked guar gum at the gel point. Food Hydrocolloids, 23, 210-220 (2009).

DOI: 10.1016/j.foodhyd.2008.01.001

[27] Pezron E., Ricard A., Leibler L.: Rheology of galactomannan-borax gels. Journal of Polymer Science Part B: Polymer Physics, 28, 2445-2461 (1990).

DOI: $10.1002 /$ polb.1990.090281301

[28] Bocchinfuso G., Mazzuca C., Sandolo C., Margheritelli S., Alhaique F., Coviello T., Palleschi A.: Guar gum and Scleroglucan interactions with borax: Experimental and theoretical studies of an unexpected similarity. The Journal of Physical Chemistry B, 114, 1305913068 (2010).

DOI: $10.1021 / \mathrm{jp} 105838 \mathrm{t}$

[29] Kök M. S., Hill S. E., Mitchell J. R.: Viscosity of galactomannans during high temperature processing: Influence of degradation and solubilisation. Food Hydrocolloid, 13, 535-542 (1999).

DOI: $10.1016 / \mathrm{S} 0268-005 X(99) 00040-5$

[30] Lapasin R., Pricl S.: Rheology of industrial polysaccharides: Theory and applications. Blackie Academic and Professional, London (1995).

[31] Kuijpers A. J., Engbers G. H. M., Feijen J., De Smedt S. C., Meyvis T. K. L., Demeester J., Krijgsveld J., Zaat S. A. J., Dankert J.: Characterization of the network structure of carbodiimide cross-linked gelatin gels. Macromolecules, 32, 3325-3334 (1999). DOI: $10.1021 / \mathrm{ma981929v}$

[32] Grassi M., Lapasin R., Pricl S.: A study of the rheological behavior of scleroglucan weak gel systems. Carbohydrate Polymers, 29, 169-181 (1996). DOI: 10.1016/0144-8617(95)00120-4

[33] Draper N. R., Smith H.: Applied regression analysis. Wiley, New York (1966).

[34] Flory P. J.: Principles of polymer chemistry. Cornell University Press, Ithaca (1953).

[35] Pasut E., Toffanin R., Voinovich D., Pedersini C., Murano E., Grassi M.: Mechanical and diffusive properties of homogeneous alginate gels in form of particles and cylinders. Journal of Biomedical Materials Research Part A, 87, 808-818 (2008).

DOI: $10.1002 / \mathrm{jbm} . \mathrm{a} .31680$

[36] Schurz J.: Rheology of polymer solutions of the network type. Progress in Polymer Science, 16, 1-53 (1991).

DOI: 10.1016/0079-6700(91)90006-7 
[37] Turco G., Donati I., Grassi M., Marchioli G., Lapasin R., Paoletti S.: Mechanical spectroscopy and relaxometry on alginate hydrogels: A comparative analysis for structural characterization and network mesh size determination. Biomacromolecules, 12, 1272-1282 (2011). DOI: $10.1021 / \mathrm{bm} 101556 \mathrm{~m}$

[38] Uh J., Watson A. T.: Nuclear magnetic resonance determination of surface relaxivity in permeable media. Industrial and Engineering Chemistry Research, 43, 3026-3032 (2004).

DOI: $10.1021 / \mathrm{ie} 030599 \mathrm{~m}$

[39] Chui M. M., Phillips R. J., McCarthy M. J.: Measurement of the porous microstructure of hydrogels by nuclear magnetic resonance. Journal of Colloid and Interface Science, 174, 336-344 (1995).

DOI: $\underline{10.1006 / \text { jcis. } 1995.1399}$

[40] Brownstein K. R., Tarr C. E.: Importance of classical diffusion in NMR studies of water in biological cells. Physical Review A, 19, 2446-2453 (1979).

DOI: 10.1103/PhysRevA.19.2446

[41] Scherer G. W.: Hydraulic radius and mesh size of gels. Journal of Sol-Gel Science and Technology, 1, 285291 (1994).

DOI: $10.1007 / \mathrm{BF} 00486171$

[42] Grassi M., Fiorentino S., Farra R., Dapas B., Grassi G.: Hydrogels mesh size evaluation. in: 'Polysaccharide hydrogels: Characterization and biomedical applications' (eds.: Matricardi P., Alhaique F., Coviello T.) Pan Stanford Publishing, Singapore, in press (2013).

[43] Dubois M., Gilles K. A., Hamilton J. K., Rebers P. A., Smith F.: Colorimetric method for determination of sugars and related substances. Analytical Chemistry, 28, 350-356 (1956).

DOI: $10.1021 / \mathrm{ac} 60111 \mathrm{a} 017$
[44] Grassi M., Lapasin R., Coviello T., Matricardi P., Di Meo C., Alhaique F.: Scleroglucan/borax/drug hydrogels: Structure characterisation by means of rheological and diffusion experiments. Carbohydrate Polymers, 78, 377-383 (2009).

DOI: $10.1016 /$ j.carbpol.2009.04.025

[45] Grassi M., Grassi G., Lapasin R., Colombo I.: Understanding drug release and absorption mechanisms: A physical and mathematical approach. CRC Press, Boca Raton (2007).

[46] Patankar S. V.: Numerical heat transfer and fluid flow. Hemisphere Publishing, New York (1990).

[47] Peppas N. A., Bures P., Leobandung W., Ichikawa H.: Hydrogels in pharmaceutical formulations. European Journal of Pharmaceutics and Biopharmaceutics, 50, 27-46 (2000). DOI: 10.1016/S0939-6411(00)00090-4

[48] Palleschi A., Bocchinfuso G., Coviello T., Alhaique F.: Molecular dynamics investigations of the polysaccharide scleroglucan: First study on the triple helix structure. Carbohydrate Research, 340, 2154-2162 (2005). DOI: $10.1016 /$ j.carres.2005.06.026

[49] Tomić K., Veeman W. S., Boerakker M., Litvinov V. M., Dias A. A.: Lateral and rotational mobility of some drug molecules in a poly(ethylene glycol) diacrylate hydrogel and the effect of drug-cyclodextrin complexation. Journal of Pharmaceutical Sciences, 97, 32453256 (2008).

DOI: $10.1002 / j p s .21251$

[50] Holz M., Heil S. R., Sacco A.: Temperature-dependent self-diffusion coefficients of water and six selected molecular liquids for calibration in accurate ${ }^{1} \mathrm{H}$ NMR PFG measurements. Physical Chemistry Chemical Physics, 2, 4740-4742 (2000). DOI: $10.1039 / \mathrm{B} 005319 \mathrm{H}$ 